\title{
SOIL-STRUCTURE INTERACTION MODELING FOR THE DYNAMIC ANALYSIS OF CONCRETE GRAVITY DAMS
}

\author{
A. De Falco ${ }^{1}$, M. Mori ${ }^{2}$, and G. Sevieri ${ }^{2}$ \\ ${ }^{1}$ Dept. of Energy, Systems, Territory and Constructions Engineering, Pisa University, Largo Lucio \\ Lazzarino, 1 - 56122 Pisa (Italy) \\ a.defalco@ing.unipi.it \\ ${ }^{2}$ Dept. of Energy, Systems, Territory and Constructions Engineering, Pisa University, Largo Lucio \\ Lazzarino, 1 - 56122 Pisa (Italy) \\ morimatteo123@gmail.com \\ ${ }^{2}$ Dept. of Civil and Industrial Engineering, Pisa University, Largo Lucio \\ Lazzarino, 1 - 56122 Pisa (Italy) \\ giacomo.sevieri@unifi.it
}

\begin{abstract}
During earthquake shaking, the dam-reservoir-foundation system has to be considered a coupled system. In this paper Soil-Structure Interaction (SSI) effect is investigated on a $2 D$ plane model of a concrete gravity dam under earthquake excitation. Firstly, different approaches to simulate the unboundedness of soil domain are explored: the Perfectly Matched Layer (PML) technique, the Low Reflecting Boundary (LRB) condition and the Infinite Elements (IEs). Different options are compared in the time domain in the case of linear elastic material. The importance of taking into account the SSI in the seismic assessment of concrete dams is also highlighted by the energy balance during time. Successively, the effects of SSI are analysed on a full interacting nonlinear plane model. The results which are obtained in terms of material damage and dissipated energy through a parametric SSI simulation in the time domain show the importance of the choice of the damage constitutive law of the material.
\end{abstract}

Keywords: Gravity dams, Soil Structure Interaction, Perfectly Matched Layer, Low Reflecting Boundary, Infinite Elements, Time history analysis. 


\section{INTRODUCTION}

The soil effects on the seismic behavior of buildings are seldom explicitly taken into account in finite element models. More often, in order to overcome the difficulties involved in modeling soil-structure interaction, code-provided response spectra depending on suitable soil categories are used. The particular characteristics of the retaining structures do not allow us to use the simplified methods which are commonly applied to ordinary buildings, so dam earthquake safety assessment requires the direct modeling of soil structure interaction.

Soil Structure Interaction is described to have two main components: kinematic and inertial interaction [1]. In the literature two contributions are relevant in approaching the problem of soil-structure interaction, those by Wilson [2] and Wolf [1]. Wilson describes the "massless foundation" method, basing on the consideration that the recorded ground motions are acquired at the terrain surface where the response has already experienced the effects of the soil. This model is governed by soil flexibility. The massless foundation model proposed by Clough in 1980 [3] has been extensively used in seismic analysis of dam-foundation problems. In this context, recorded displacements are imposed at the boundaries of the domain and the input motion reaches instantaneously the base of the dam disregarding the inertial interaction. In the massless model, the wave velocity in foundation becomes infinite, so the input motion reaches instantaneously the base of the dam and the structure takes all kinetic energy. These assumptions seem in general unrealistic [4].

Inertial interaction is generated by elastic waves that develop under dynamic loads, promoting the energy transport through the soil volume. Such a phenomenon that carries energy away from the structure is often referred as "radiation damping". So, while in static SSI analysis the simple truncation of the far field with setting of appropriate boundary conditions gives very often good results, in dynamic cases it makes results to be erroneous because of reflection waves.

The present paper addresses Soil-Structure Interaction (SSI) for existing concrete gravity dams, investigating its effects numerically on a 2D plane system under earthquake excitation. In order to simulate the unboundedness of soil domain, different modelling approaches are explored: the Perfectly Matched Layer (PML) technique [5], the Low Reflecting Boundary (LRB) condition [6] and the Infinite Elements (IEs) [7]. Moreover, the analysis in the time domain is performed in order to compare the response of the model in terms of base shear, using different modelling options. The energy balance during time shows the importance of modeling the SSI in the seismic assessment of concrete dams. The results in terms of base shear values for several modeling approaches are compared in order to highlight the differences among different modelling strategies.

Finally the effects of SSI are analysed on a full interacting nonlinear plane model with nonlinear material constitutive behaviour. The results in terms of material damage are obtained from a parametric SSI simulation in the time domain, in order to take into account the presence of soil beneath the dam.

The main contribution of this work is to compare different modeling strategies and to highlight the importance of SSI modelling for the evaluation of the seismic behaviour of concrete gravity dams.

\section{MODELLING UNBOUNDED SOIL}

Dam models seldom take into account full interaction effects, because of the lack of adequate numerical implementations or computational resources required by three dimensional detailed models. Recently, SSI for concrete retaining structures is addressed by many authors searching for a reliable simulation of wave propagation in a semi-infinite medium, modeling 
the far field part of the foundation. The unboundedness of the terrain was first considered by Lamb [8] in its classical problem of a point load on a half space, for which he provided an analytical solution. Wolf [4], on the other hand, developed a formulation for appropriate springdashpot coefficients and boundary conditions. Some worth noting methods are Lysmer boundary conditions [6], hyperelements [9], infinite elements [10], [11], rational boundary conditions [12], boundary element method [13], scaled boundary element method [14] and high order non-reflecting boundary conditions [15].

In order to simulate the unboundedness of both solid and fluid domains, three different modeling options are explored in this work, the Perfectly Matched Layer (PML) technique, the Low Reflecting Boundary (LRB) condition and the Infinite Elements (IEs). PML have been widely used for simulating wave propagation in unbounded media to effectively avoid spurious wave reflections from the computational domain boundaries. This technique is able to absorb incident waves under any angle and frequency, preventing them from returning back to the medium after incidence to the model boundaries [16]. The procedure, which was first introduced by Berenger in 1994 [5], may be applied to different physical problems. It comes to a complex coordinate stretching of the domain to introduce a decay of the oscillation avoiding any reflection in the source domain, thus simulating a perfectly absorbing material. The rational scaling of PML is expressed by the following function of the dimensionless coordinate $\xi[16]$

$$
f(\xi)=s \cdot \xi\left(\frac{1}{3 p(1-\xi)+4}-\frac{i}{3 p(1-\xi)}\right)
$$

where $p$ is the curvature parameter and s the scaling parameter.

Implementation of PML into most commercial FE software packages is performed only in the frequency domain, because the majority of the formulations in the time domain highly affect the computational time and resources.

IEs, which are used to incorporate unbounded domains into the finite element method, have a formulation similar to those of FEM, except for the infinite extent of the element region and shape function in one direction. IEs is based on a function which maps the global to the local coordinate system

$$
f(\xi)=\frac{\xi}{\gamma-\xi} \Delta p
$$

where $\Delta p$ is the pole distance,

$$
\gamma=\frac{\Delta s+\Delta p}{\Delta s}
$$

and $\Delta s$ is the scaled thickness [7].

Finally, the LRB condition is obtained by imposing a mechanical impedance on the foundation boundary of the model, following the equation

$$
[T] \cdot n=-[D] \frac{d u}{d t}
$$

where $u$ is the displacement vector, $[T]$ the stress tensor, $n$ the unit vector of the boundary tangent plane, $[D]$ is the impedance matrix [17] 


$$
[D]=\frac{c_{p}+c_{s}}{2}[\mathbf{I}], \quad c_{s}=\sqrt{\frac{G}{\rho}}, \quad c_{p}=\sqrt{\frac{K+4 / 3 G}{\rho}}
$$

where $K$ is the bulk modulus of the soil, $G$ the shear modulus, $\rho$ the material's density and $[\mathrm{I}]$ the identity matrix.

\section{A COMPARISON AMONG SSI MODELING STRATEGIES}

Different modeling strategies are analyzed and compared in terms of resultant base shear force of a 2D plane strain system under earthquake excitation. The analyses are carried out in the time domain using COMSOL Multiphysics [18].

The dam and soil domains are modeled by applying the standard Solid Mechanics equations. Three different techniques which have been discussed above are used to simulate the unboundedness of the soil: the Perfectly Matched Layer (PML) technique, the Low Reflecting Boundary (LRB) condition and the Infinite Elements (IEs). The study in the time domain is performed on an Italian concrete gravity dam 65 meters tall, with a base of about $45 \mathrm{~m}$, using four plane strain models, as shown in figure 1.

The reference model 1 simulates the dam on a rigid terrain. The solid mesh is composed by 453 default second-order serendipity elements and the displacements of the base nodes are restrained along both directions.

In this case, the basin was simulated by the added mass model [19].

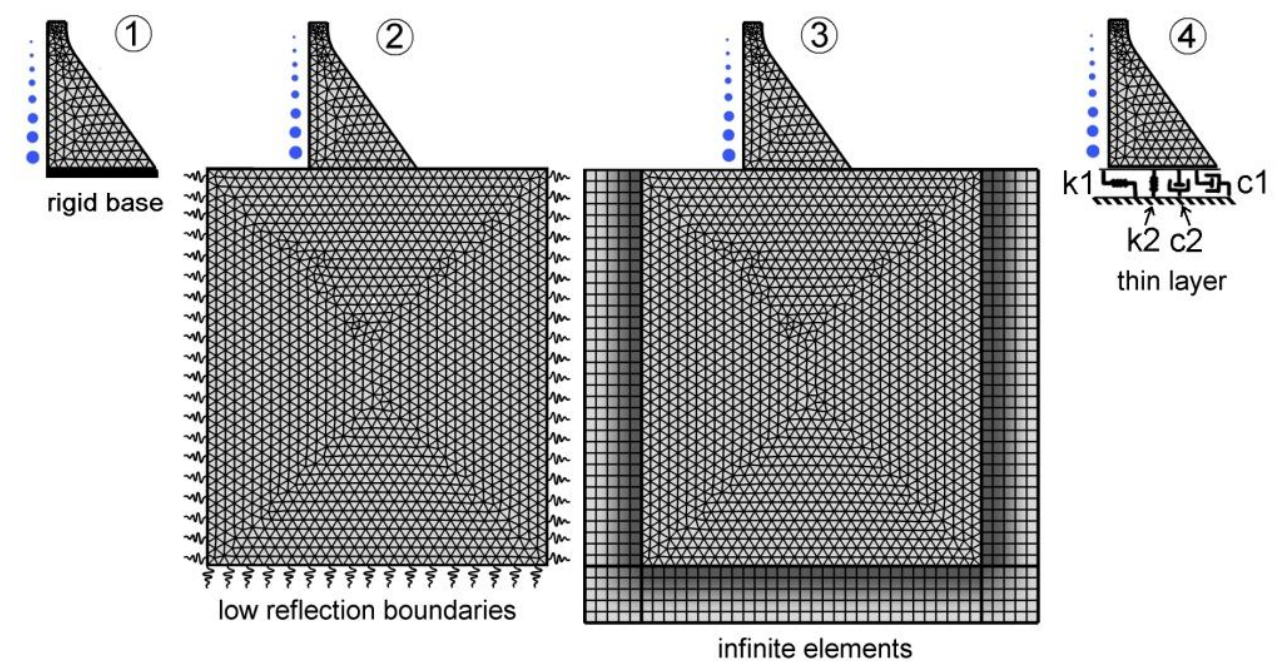

Figure 1. Different SSI modeling strategies for a plane strain model of an Italian dam.

The other three models account for massed foundation as an unbounded half-space.

The second model simulates the dam with the soil equipped by low reflection boundaries. The solid mesh is composed by 3146 default second-order serendipity elements for both the soil and the dam. The low reflecting boundary condition is defined on the boundary of the soil domain. Its impedance parameter values derive from the elastic properties of the soil domain. The third model is similar to the second one, but in addition there are 550 infinite elements surrounding the physical soil region. The solid mesh is thus composed by 3696 default second-order serendipity elements. Infinite element pole distance is $400 \mathrm{~m}$ and the scaled thickness is $10^{3} \cdot 400 \mathrm{~m}$.

The fourth model of fig. 1 simulates the unboundedness of the soil through the PMLs applied at the bottom and on the sides. Unfortunately, PML technique is not always available in 
each program code or, if present, may not be compatible with time domain analyses. For this reason, a suitable 2D simplified model with horizontal and vertical springs and dashpots at the base was created in [20] to take into account the SSI by using tools and standard boundary conditions. Two viscous damping coefficients and two spring stiffness coefficients were calibrated in [20], starting from a model which is similar to that of case 2 of figure 1 , but is equipped by PML boundary elements around the soil domain, instead of low reflecting boundaries. Calibration has been performed to provide the same resultant shear force at the base of the dam in the frequency domain for both models: a simplified model and the interacting 2D model with PML boundary condition. The result is that there is a set of coefficients which are capable to simulate SSI in terms of resultant shear force at the base of the dam. Their values are independent from the frequency in the range between $0 \mathrm{~Hz}$ and $25 \mathrm{~Hz}$ with good approximation and they are reported in table 1 .

Table 1: Resulting parameters of the simplified model approximating the PML boundary technique.

\begin{tabular}{|c|c|c|}
\hline parameter & {$[\mathrm{N} / \mathrm{sqm}]$} & {$[\mathrm{N} \cdot \mathrm{s} / \mathrm{sqm}]$} \\
\hline $\mathrm{k}_{1}$ & $\begin{array}{l}8,6 \mathrm{e}+9 \pm \\
1,1 \mathrm{e}+9\end{array}$ & \\
\hline \multirow[t]{3}{*}{$\mathrm{k}_{2}$} & $\begin{array}{l}9,7 \mathrm{e}+8 \pm \\
9,3 \mathrm{e}+7\end{array}$ & \\
\hline & & $3,6 e+7 \pm 2,3 e+6$ \\
\hline & & $1.5 \mathrm{e}+7 \pm 6,8 \mathrm{e}+6$ \\
\hline
\end{tabular}

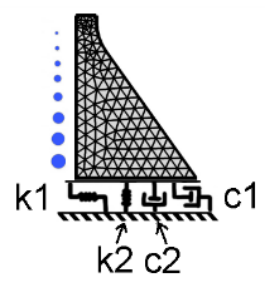

The four models share the same plane-strain setting and material properties, whose values are reported in Table 2.

Table 2. Material properties which are assumed for the comparison among SSI modelling strategies.

\begin{tabular}{lll}
\hline Material property value & Concrete & Foundation rock \\
\hline Density $\boldsymbol{\rho}\left(\mathrm{kg} / \mathrm{m}^{3}\right)$ & 2450 & 2300 \\
Young modulus $\mathbf{E}(\mathrm{MPa})$ & 20500 & 22000 \\
Poisson modulus v & 0.2 & 0.2 \\
Damping coefficient $\boldsymbol{\xi}$ & 0.05 & 0.05 \\
\hline
\end{tabular}

In order to perform the analysis in the time domain for the fourth model of fig. 1, the Thin Elastic Layer boundary condition of COMSOL is applied at the base of the dam. It has both elastic and damping properties and can model a thin elastic layer with specified stiffness and damping properties.

The dynamic excitation which was used in the model belongs to one of the Italian strongest events occurred in the last 30 years, the earthquake of Central Italy of October 30th 2016 (06:40:17 UTC, $6.5 \mathrm{MW})$. It is the E-W component with about $7.8 \mathrm{~m} / \mathrm{sqs}$ PGA, which was recorded by Savelli (PG) station [21] (Fig. 2). The duration of transient load is $30 \mathrm{~s}$ and the sampling is $5 / 1000 \mathrm{~s}$.

The analysis has been carried out by assigning the seismic shaking in form of volume loads to the dam domain, in addition to the self-weight and the hydrostatic load. The results in form of base shear are reported in Fig. 3, where the graphs are overlapped. The similarity between the results obtained with the different modelling strategies for the semi-finite soil is remarkable, while a certain difference between these and the rigid case is noteworthy, although the heights of some main peaks remain quite unchanged. 


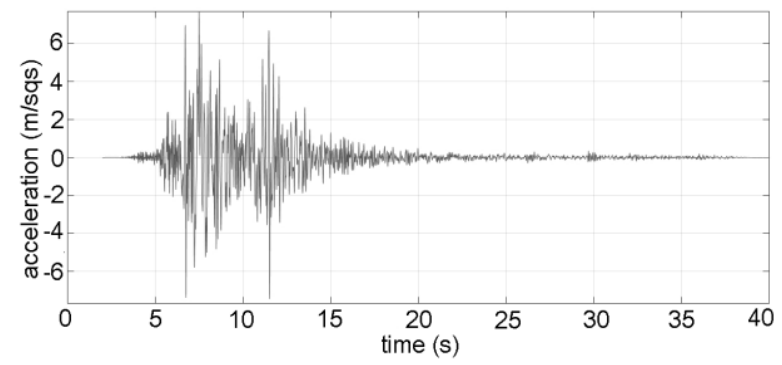

Figure 2. The acceleration time history recorded on October $30^{\text {th }} 2016$ by Savelli station (record 1).

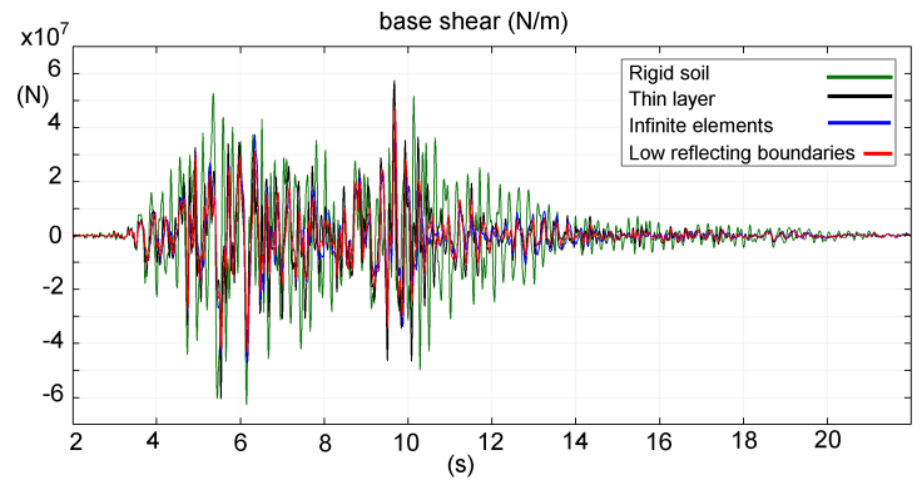

Figure 3. Base shear resultant force obtained from the transient analysis for the four different modeling options.

The FFT of the signal of Fig. 3 is reported in figure 4. The FFT graphs for the different modeling approaches substantially coincide except for the amplitude of some peaks, while, as expected, the graph of the rigid case shows higher peak frequencies and relative peak values.

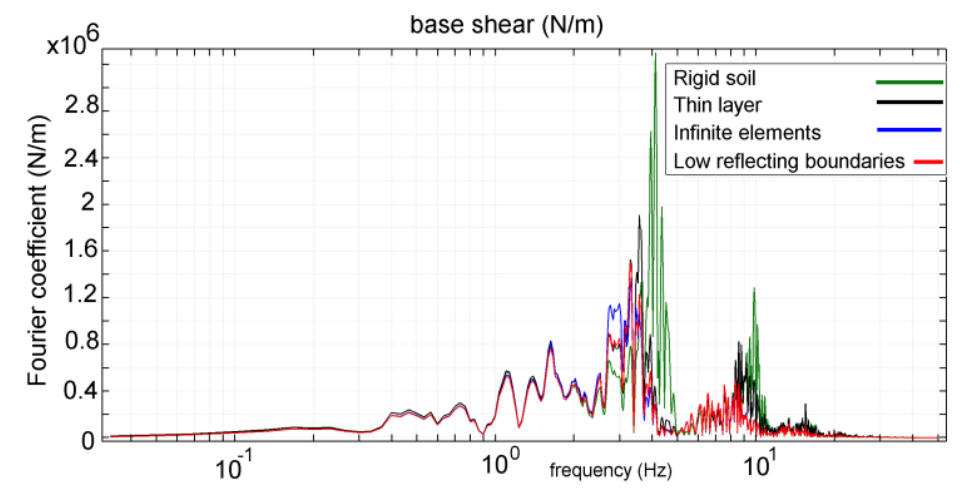

Figure 4. FFT of the base shear history for the different SSI modeling approaches.

Finally, in Fig. 5 the Root Mean Square value of the resultant base shear for different modeling options is displayed. Also this time, it may be observed that RMS values for the cases of thin layer, low reflection boundary and infinite elements are very similar to each other, while they substantially differ from the rigid base case. In addition, the models with IEs and LRB almost coincide.

The graph of the energy balance during the time history analysis on the model with LRB is displayed in Fig. 6. One can note that the most part of dissipated energy is radiated and the energy dissipated within both dam and soil material is about $45 \%$ of the radiated one. In its turn, the energy dissipated within the dam material only is about $70 \%$ of the energy dissipated within the solid domain. The contribution of radiation damping is therefore a no-negligible part of the total dissipated energy. 


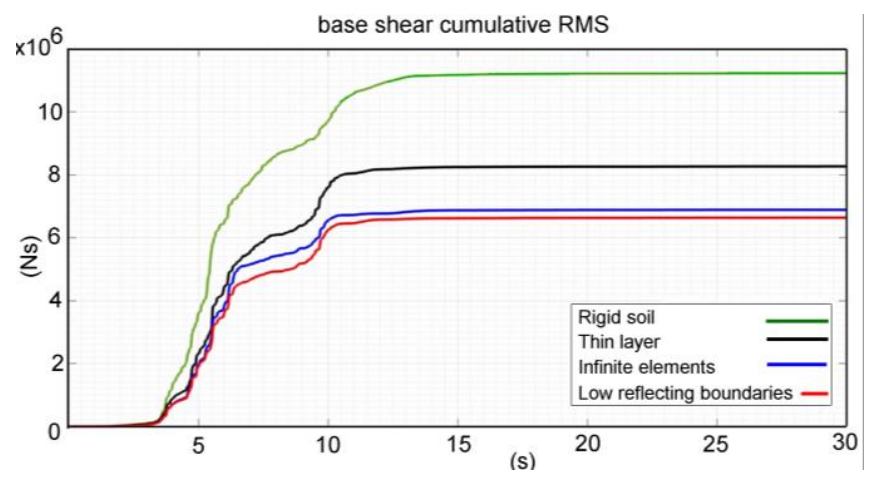

Figure 5. Base shear Root Mean Square value for different modeling options.

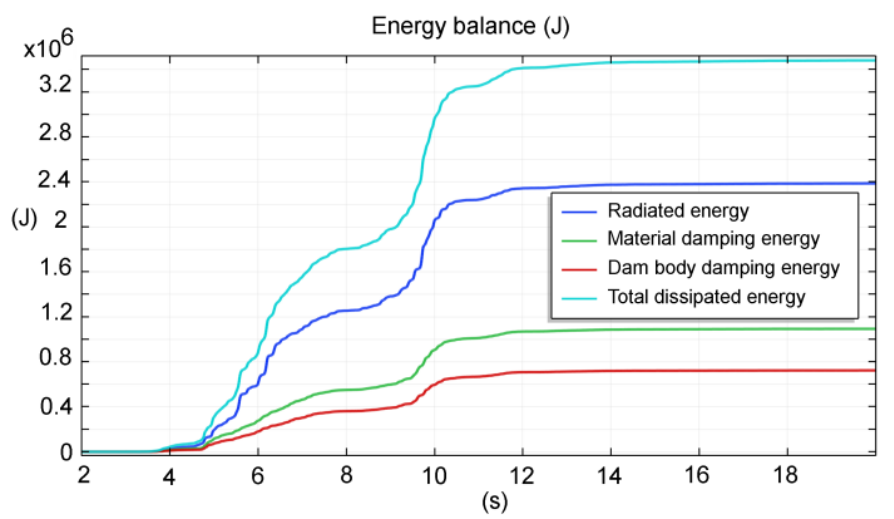

Figure 6. Energy balance (J) during the time history analysis for the LRB model.

\section{SSI IN NONLINEAR 2D MODELS}

In order to evaluate the effects of taking into account SSI in a more detailed nonlinear model, a 2D full interacting model was created in Abaqus r.6.14 [22]. It simulates a dam $55 \mathrm{~m}$ tall, with a base length of $40 \mathrm{~m}$, in plane stress state. The soil domain dimensions are $260 \mathrm{~m} \mathrm{x}$ $110 \mathrm{~m}$ and the reservoir level is $53 \mathrm{~m}$ high. The thickness of the plane model is $1 \mathrm{~m}$.

The standard Solid Mechanics equations are applied to the dam and soil domains. The solid mesh is composed by 774 linear quadrilateral elements CPS4R for the soil domain and by 1149 linear triangular elements CPS3 for the dam domain. 79 infinite elements CINPS4 surrounding the soil domain allow one to simulate the soil unboundedness.

The fluid subsystem is simulated by the Helmoltz equation derived from the full NavierStokes equation, assuming small vibrations and neglecting viscosity. The mesh of the fluid domain is composed by 2912 linear triangular elements AC2D3.

As for the boundary condition for the fluid domain, a zero pressure condition is imposed at the free surface of the water and a rigid wall condition at the bottom of the reservoir. The fluid-structural interaction condition at the interface between the two domains is the following

$$
\left\{\begin{array}{c}
-\boldsymbol{n}\left(\frac{1}{\rho} \nabla \sigma\right)=-\boldsymbol{n} \cdot \boldsymbol{u}_{t t} \\
F_{A}=\sigma \cdot \boldsymbol{n}
\end{array}\right.
$$

where $\sigma$ is the fluid pressure, $\boldsymbol{n}$ is the normal direction to the interface, $F_{A}$ is the acoustic force on the structure and $\boldsymbol{u}_{t t}$ is the solid acceleration. The first equation transfers the structural acceleration to the fluid, while the second applies the fluid pressure load on the structure. Such a 
system of equations expresses a fully coupled problem, where the solid and the acoustic parts have to be solved simultaneously.

In order to simulate the unboundedness of the fluid domain, an acoustic impedence in form of Improved Planar nonreflecting boundary condition was introduced at the upstream side of the fluid domain. The total number of elements within the model is 4914 , whereas the total number of nodes is 3170 .

The constitutive model selected for the dam is the Concrete Plastic Damage Model (CPDM) following the formulation proposed by Lee and Fenves [23], which uses the concepts of fracture-energy-based damage and stiffness degradation in continuum damage mechanics. In order to avoid mesh-dependency problem when simulating damage, a constitutive law formulation in terms of displacements was selected. The values of material properties are reported in table 2 and the parameters of the CPDM implementation in Abaqus [22] are reported in table 3 [24].

The seismic shaking of figure 1 was applied in form of displacement to 12 nodes of the base contour of the solid domain, once it was reduced with a scale factor of 0.6.

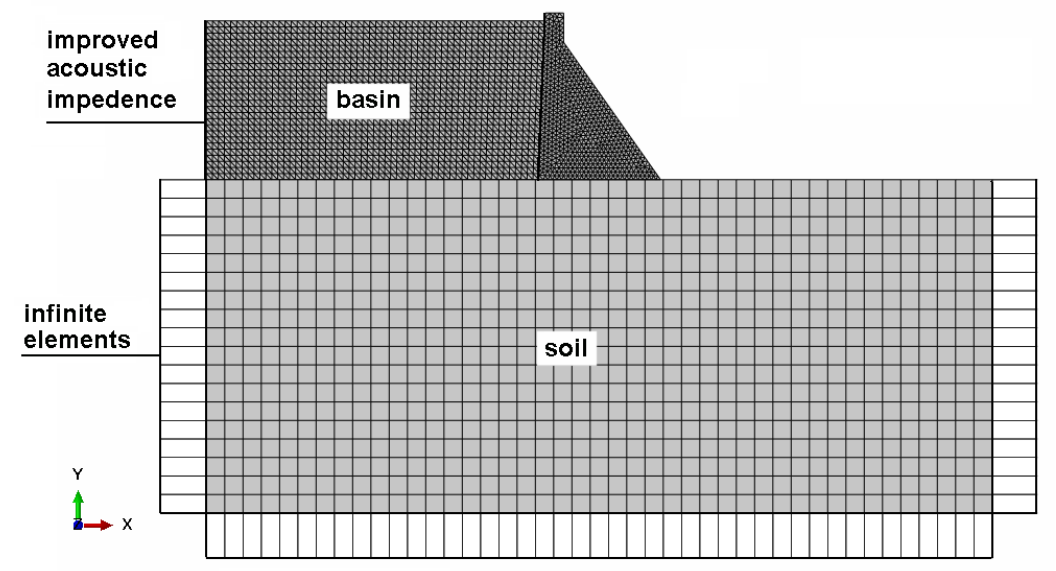

Figure 7. The model for nonlinear time history analysis.

Table 3. Parameters of the CPDM [22].

\begin{tabular}{ll}
\hline parameters for the concrete & \\
\hline Dilation angle $\left(^{\circ}\right)$ & 32 \\
Flow potential eccentricity $\varepsilon$ & 0.1 \\
$\sigma_{b 0} / \sigma_{c 0}$ ratio of initial equibiaxial compressive yield stress to initial uniaxial & 1.16 \\
compressive yield stress & \\
Shape factor Kc & 0.66 \\
Compressive strength $(\mathrm{MPa})$ & 17 \\
Tensile strength $(\mathrm{MPa})$ & 1 \\
Fracture energy $(\mathrm{Nm})$ & 150 \\
\hline
\end{tabular}

In order to apply the recorded seismic shaking to the base of the soil model, the deconvolution of the signal was performed through the algorithm of Sooch and Bagchi [24].

As a first evaluation on the SSI effects, one can wonder whether the interaction forces are able to change the basement motion as compared to the free-field ground motion (i.e. motion recorded on the free surface of the soil without structure). To this aim, in Fig. 8 the acceleration of a point on the soil surface near to the dam base (red dashed line) has been compared with the acceleration of the same point in a model that does not include the dam (black continuous line). In Fig. 9 the FFT of both signals is reported. One can note that the peak acceleration is higher in the free-field model. The FFT shows that the greatest amplifications of the 
free-field model in respect to the model with dam take place in the interval between $5 \mathrm{~Hz}$ and $10 \mathrm{~Hz}$, although the peak frequencies remain almost unchanged. It can be concluded that the interaction forces are able to considerably change the soil motion in this case.

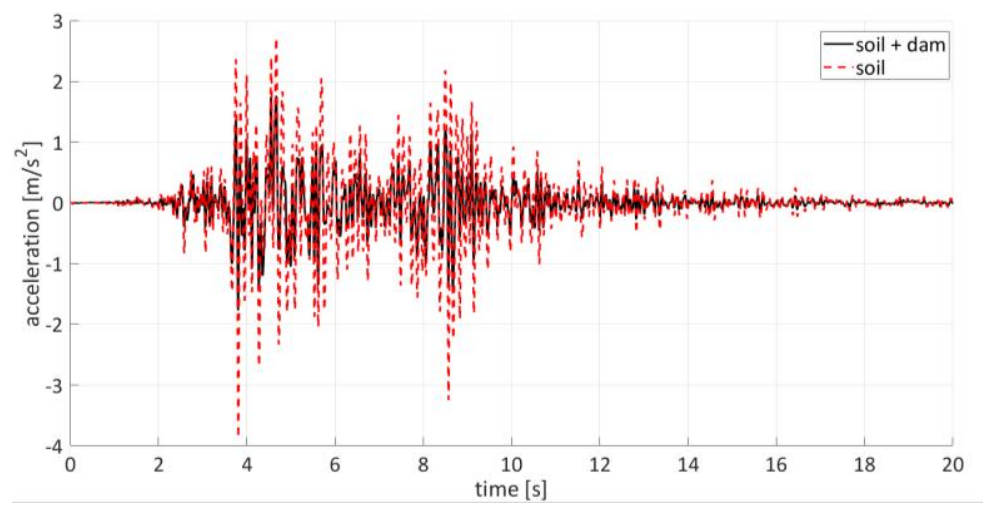

Figure 8. Acceleration history on a point of the soil surface for the model including the dam (red dashed line) and for the free-field model (black continuous line).

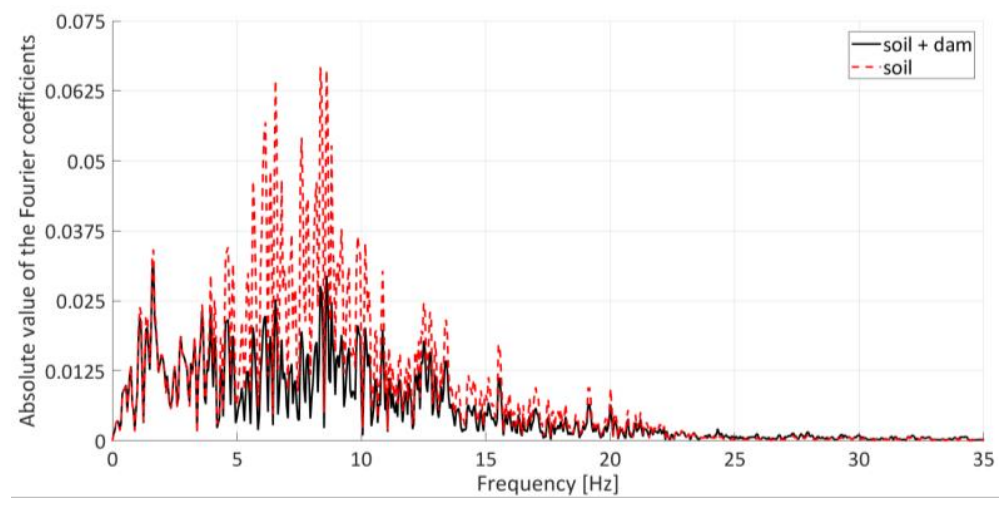

Figure 9. FFT of the signals of Fig. 8.

Another issue concerns the damaging mode of the dam after shaking when CPDM is used to simulate the material behaviour. In this regard, the influence of both soil density and damping ratio variation was investigated and the effects of different acceleration records applied to the model were explored.

As soil density seems to be a key parameter for SSI effects [20], it was varied in order to evaluate the damaged state of the dam after shaking. In Figure 10 the damage level after the seismic sequence is presented. The legend reports the values of the complement to unity of the ratio between damaged deformability modulus and undamaged one. Cracks begin on the upstream side and at the interface between dam and soil and develop downstream across the dam body. Two different soil density values are considered: the first is greater than the actual density of an order of magnitude and the second is near to zero. Figure 10 reports the damaged areas in the two extreme cases in comparison with the case with actual density. Despite the considerable variation in the soil density value, the influence in terms of material damage is not significant. One can conclude that there are no effects on the type and extent of damage as a result of the different or incorrect appreciation of the soil density value.

The influence of viscous damping can be evaluated for the full interacting nonlinear system in term of dissipated energy. Two different values for damping ratio were considered: $\xi=1 \%$ and $\xi=5 \%$. The energy balance was calculated in both cases. In figure 11 the dam body damping dissipated energy versus time is represented by a red line, the plasticity dissipated energy is indicated by a yellow line and the damage dissipated energy by a blue line. 
One can note that in both cases the dissipated energy due to plasticity is of the same order of magnitude as the dam body damping energy, while material damage dissipates a very low energy amount in respect to the other two quantities.

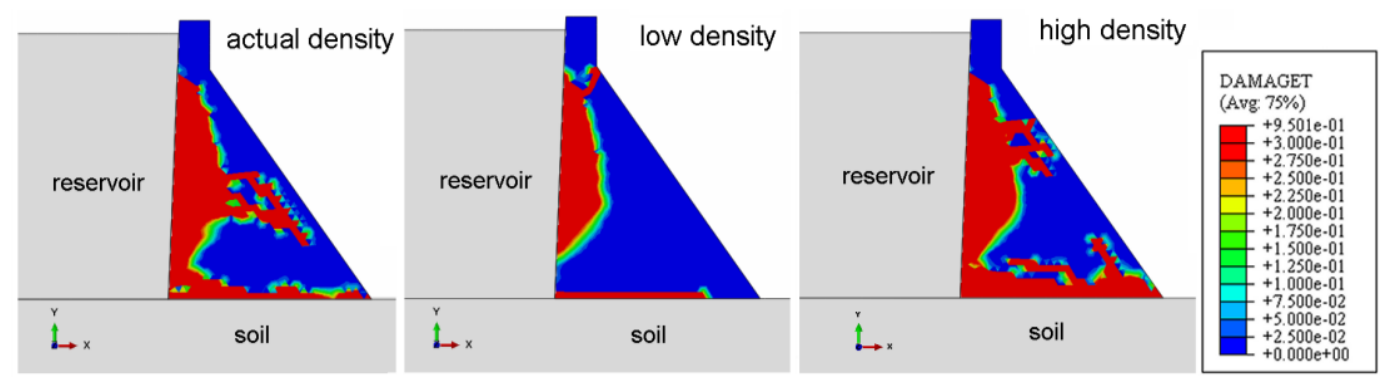

Figure 10. Damage on the dam, varying density.
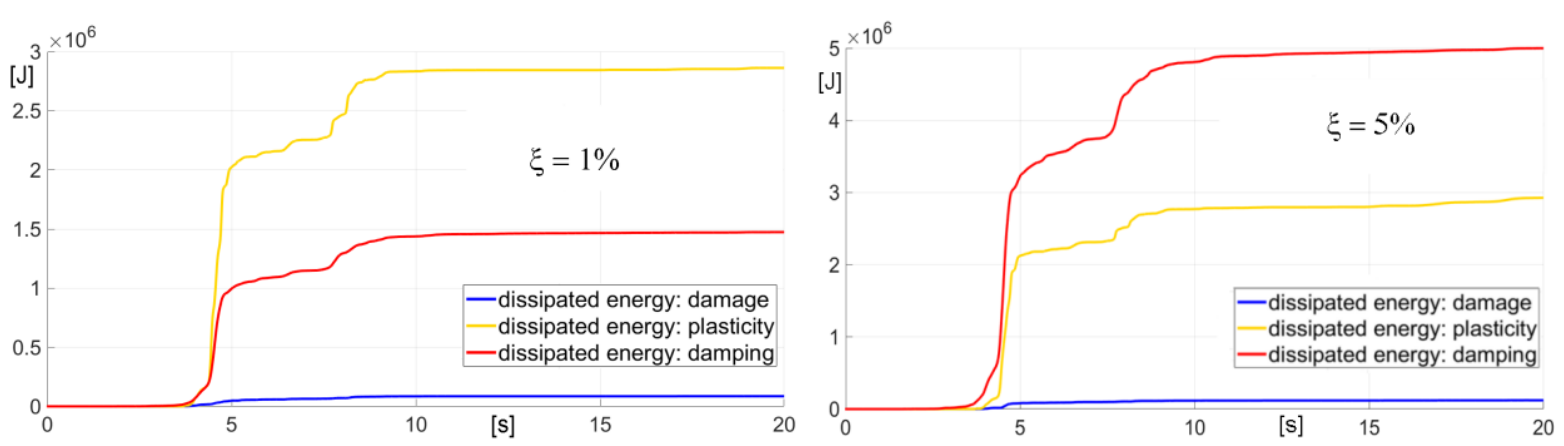

Figure 11: Dissipated energy for damage, plasticity and dam body damping for different values of $\xi$.

The effect of the type of seismic shaking was evaluated, in its turn, by comparing the damaged state after the shaking of October 30th 2016 (record 1) with that after the shaking of August $24^{\text {th }} 2016$ (record 2) in Central Italy (Fig. 12).

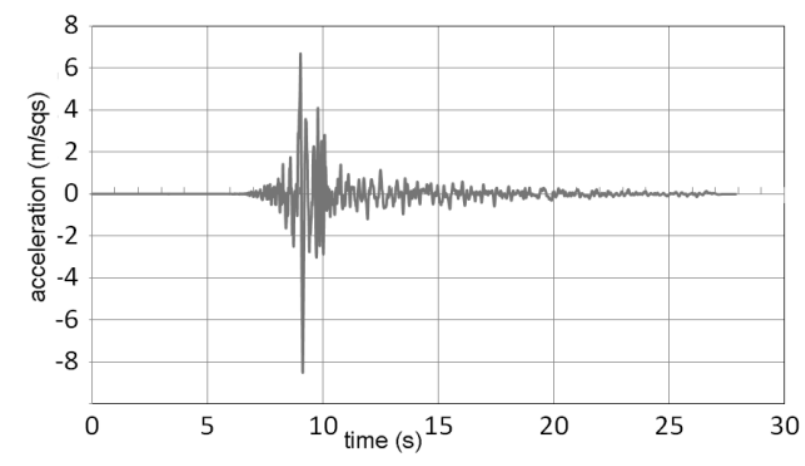

Figure 12. The acceleration time history recorded on August $24^{\text {th }} 2016$ by Amatrice station (record 2).

This latter, belonging to the seismic sequence of August 24th 2016 (03:36:32 UTC, 6 MW), is the E-W component recorded by Amatrice (RI) station [21] with about $8.5 \mathrm{~m} / \mathrm{sqs}$ PGA. As in the previous case, it was reduced by 0.6 before being applied.

Resulting damage of the dam is displayed in figure 13 for both dynamic excitations applied at the base of the soil domain. For each record, the initial and final damage state are reported. The damaged state is different in the two cases both in the initial and in the final phase. In the case of record 1 and tensile strength of $1 \mathrm{MPa}$, cracks initiate from the upstream side of the dam, whereas in the second case, they initiate from the downstream side. Damage evolves in a very different way, as can be seen from the figures of final damage. 


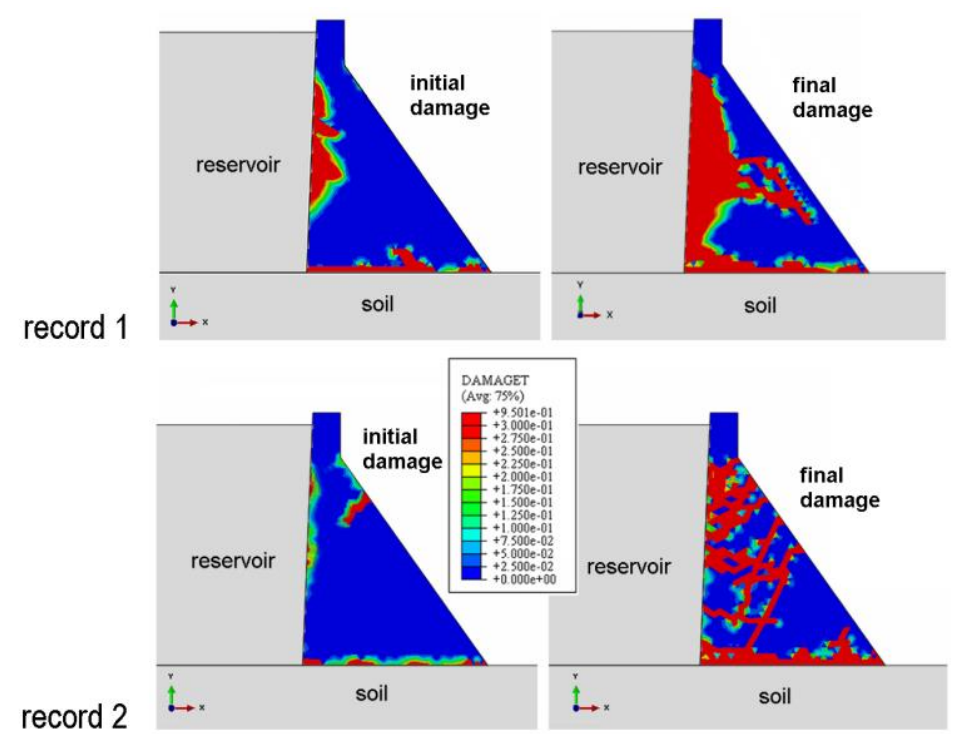

Figure 13: Damage of the dam during the shaking of October 30th 2016 (record 1) and August 24th 2016 (record 2).

\section{CONCLUSIONS}

We can conclude that different modelling strategies for simulating the soil unboundedness provide very similar results in terms of base shear force and dissipated energy. Moreover, the most part of dissipated energy is the radiated one, thus, the simulation of the unbounded soil appears very important.

When the material is nonlinear, the amount of dissipated energy due to plastic deformation has the same order of magnitude of the dissipated energy due to material damping. On the contrary, the energy that is dissipated by the material damage is particularly low. Hence, the assumption of plastic behaviour of the material could provide large consequences in terms of dissipated energy. Finally, the damaged state of the dam is not affected by the variation of soil density, nor by that of the material damping, but it is largely influenced by the frequency content of the shaking. In conclusions, in order to obtain reliable results, SSI modeling and the material damage law selection must be carefully considered.

\section{REFERENCES}

[1] J.P. Wolf, Dynamic Soil Structure Interaction. Englewood Cliffs, New Jersey, PrenticeHall, 1985.

[2] E.L. Wilson, Three-Dimensional Static and Dynamic Analysis of Structures, A Physical Approach With Emphasis on Earthquake Engineering. Computers and Structures, Inc., Berkeley, California, 2002.

[3] R.W. Clough, Non-linear mechanisms in the seismic response of arch dams. International Conference on Earthquake Engineering. Skopje, Yugoslavia, 1980.

[4] H. Tan, A.K. Chopra, Earthquake analysis of arch dams including dam-water foundation rock interaction. Earth. Eng. and Struct. Dyn., 24, 1453-1474, 1995.

[5] J.P. Berenger, A perfectly matched layer for the absorption of electromagnetic waves, $J$. Comput. Phys., 114, 185-200, 1994.

[6] J. Lysmer, R.L. Kuhlemeyer, Finite dynamic model for infinite media. J. Eng. Mech., ASCE 95, 859-878, 1969. 
[7] O.C. Zienkiewicz, C. Emson, P. Bettess, A novel boundary infinite element. Int. J. for Numerical Methods in Engineeering. 19, 1983.

[8] H. Lamb, On the Propagation of Tremors over the Surface of an Elastic Solid, Philosophical Transactions of the Royal Society of London. Series A, 203, 1-42, 1903.

[9] V. Lofti, J.L. Tassoulas, J.M. Roesset, A technique for the analysis of dams to earthquakes, Earthquake. Engineering \& Structural Dynamics. 15, 463-490, 1987.

[10] D.K. Kim, C.B. Yun, Time domain soil-structure interaction analysis in two dimensional medium based on analytical frequency-dependent infinite elements. Int. J. Numer. Meth. Eng., 47, 1241-1261, 2000.

[11] C.B. Yun, D.K. Kim, J.M. Kim, Analytical frequency-dependent infinite elements for soil-structure interaction analysis in two-dimensional medium. Engineering Structures, 22, 258-271, 2000.

[12] G. Feltrin, Absorbing boundaries for the time-domain analysis of dam-reservoirfoundation systems. Report Swiss Federal Institute of Technology Zurich, 1997.

[13] M. Yazdchi, N. Khalili, S. Valliappan, Nonlinear seismic behaviour of concrete gravity dams using coupled finite element-boundary element technique. International Journal for Numerical Methods in Engineering, 44, 101-130, 1999.

[14] C. Song, J.P. Wolf, The scaled boundary finite-element method, a primer: solution procedures. Comput. Struct., 78, 211-225, 2000.

[15] D. Givoli, High-order local non-reflecting boundary conditions: a review. Wave. Mot., 39, 319-326, 2004.

[16] S. Johnson, Notes on perfectly matched layers (PMLs). Massachusetts Institute of Technology, Tech. Rep., 2007.

[17] G.A. Holzapfel Nonlinear Solid Mechanics: A Continuum Approach for Engineering. John Wiley \& Sons, 2000.

[18] COMSOL® Multiphysics, User's Guide, Version 5.3, COMSOL, 2017.

[19] A. De Falco, M. Mori, G. Sevieri, Simplified Soil-Structure interaction models for concrete gravity dams. R. Owen, R. de Borst, J. Reese and C. Pearce Eds. 6th European Conference on Computational Mechanics (ECCM 6) 11-15 June 2018, Glasgow, UK.

[20] H. M. Westergaard, Water pressures on dams during earthquakes. Trans. ASCE, 98, 418-433, 1933.

[21] L. Luzi, R. Puglia, E. Russo \& ORFEUS WG5, Engineering Strong Motion Database, Istituto Nazionale di Geofisica e Vulcanologia, Observatories \& Research Facilities for European Seismology, doi: 10,13127/ESM, 2016.

[22] Abaqus r.6.14 Reference Guide.

[23] J. Lee, G.L Fenves, Plastic-damage concrete model for earthquake analysis of dams. Earthquake Engineering and Structural Dynamics, 27, 937-956, 1998.

[24] J. Lubliner, S. Oliver, S. Oller, E. Oñate, A plastic-damage model for concrete, International Journal of Solids and Structures, 25, 299-326, 1989.

[25] G.S. Sooch, A. Bagchi, A new Iterative procedure for deconvolution of seismic ground motion in dam-reservoir-foundation systems. Journal of Appl. Math., 2014, 2014. 